\title{
The value of quantified plaque analysis by dual-source coronary CT angiography to detect vulnerable plaques: a comparison study with intravascular ultrasound
}

\author{
Mingyuan Yuan ${ }^{1 \#}$, Hao $\mathrm{Wu}^{1 "}$, Rongxian $\mathrm{Li}^{1}$, Mengmeng $\mathrm{Yu}^{2}, \mathrm{Xu} \mathrm{Dai}^{2}$, Jiayin $\mathrm{Zhang}^{2}$ \\ ${ }^{1}$ Department of Radiology, Affiliated Zhoupu Hospital, Shanghai University of Medicine and Health Science, Shanghai 201318, China; ${ }^{2}$ Institute of \\ Diagnostic and Interventional Radiology, Shanghai Jiao Tong University Affiliated Sixth People's Hospital, Shanghai 200233, China
}

\#These authors contributed equally to this work.

Correspondence to: Jiayin Zhang. Institute of Diagnostic and Interventional Radiology, Shanghai Jiao Tong University Affiliated Sixth People's Hospital, No. 600, Yishan Rd, Shanghai 200233, China. Email: andrewssmu@msn.com.

Background: To investigate the diagnostic performance of quantified plaque analysis and high-risk
plaque characterization by coronary computed tomography angiography (CCTA) for identifying thin-cap
fibroatheroma (TCFA).

Methods: Patients who underwent both CCTA and intravascular ultrasound (IVUS) within 4 weeks were retrospectively included. CT-derived quantitative and qualitative parameters, including diameter stenosis, minimal lumen area (MLA), low attenuation plaque (LAP) volume napkin-ring sign (NRS), positive remodeling (PR) and spotty calcification, were recorded. TCFA lesions and non-TCFA lesions were determined by IVUS. Multivariate regression analysis was used to determine the independent predictors of TCFA lesions.

Results: Sixty-five patients (mean age: 69.8 99.2 years, 29 females) with 89 lesions were finally included. LAP and NRS were more frequently presented in the group of TCFA lesions. The mean LAP volume of TCFA lesions was significantly larger than that of non-TCFA lesions [16.5 (11.0-23.0) vs. $0(0-1.5) \mathrm{mm}^{3}$, $\mathrm{P}<0.001]$. According to multivariate logistic regression analysis, LAP volume was the only significant predictor for IVUS-confirmed vulnerable plaques (odds ratio $=3.294$, 95\% confidence interval: $1.177-$ 9.223, $\mathrm{P}=0.023$ ). LAP volume showed largest area under curve (AUC) for diagnosing TCFA lesions (AUC $=0.901,95 \%$ confidence interval: $0.819-0.954, \mathrm{P}<0.0001)$. When using $>8 \mathrm{~mm}^{3}$ as the best cutoff value, the diagnostic accuracy, sensitivity and specificity of LAP volume for predicting TCFA lesions were $91.0 \%$ (81/89), 84.6\% (22/26) and 96.8\% (61/63) respectively.

Conclusions: CT-derived LAP volume of TCFA lesions was significantly higher than those of non-TCFA lesions. LAP volume was the strongest predictor for TCFA lesions as validated by IVUS.

Keywords: Coronary artery disease; multidetector computed tomography; angiography; atheroma; percutaneous coronary intervention

Submitted Aug 11, 2019. Accepted for publication Jan 08, 2020.

doi: $10.21037 /$ qims.2020.01.13

View this article at: http://dx.doi.org/10.21037/qims.2020.01.13

\section{Introduction}

Coronary vulnerable plaque, also known as thin-cap fibroatheroma (TCFA), is pathologically characterized by a thin fibrous cap $(<65 \mu \mathrm{m}$ in thickness) and large necrotic core with spotty calcification and positive remodeling (PR) $(1,2)$. Plaque rupture of TCFA lesions is considered the major underlying cause of acute coronary syndrome (ACS) (3). Previous longitudinal intravascular ultrasound 


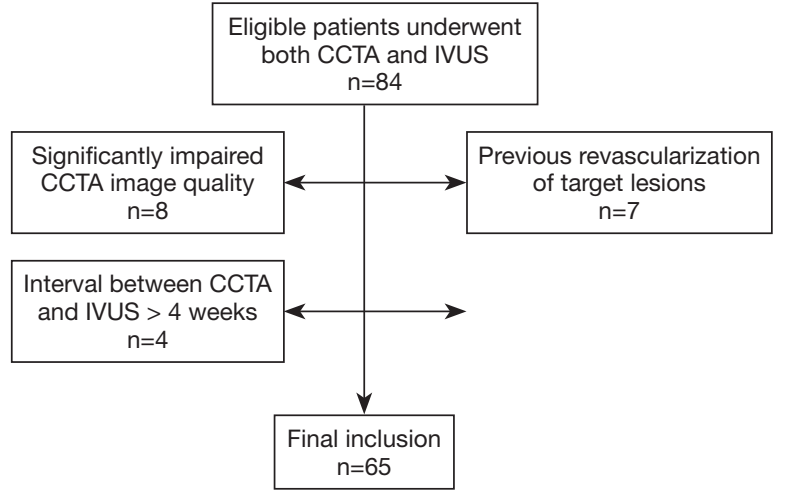

Figure 1 Flow chart of patient inclusion and exclusion criteria. CCTA, coronary computed tomography angiography; IVUS, intravascular ultrasound.

(IVUS) study also confirms that TCFA is independent predictor for major adverse cardiac events (4). However, IVUS is an invasive imaging modality and associated with approximately $1.6 \%$ of procedural related vascular injuries (4). Therefore, it cannot be indicated in all patients for the screening of vulnerable plaques. It is of clinical importance to search for another non-invasive imaging method for early detection and treatment of coronary vulnerable plaques.

Coronary computed tomography angiography (CCTA) is one wildly applied imaging modality with high sensitivity and negative predictive value (NPV) to safely rule out obstructive coronary artery disease $(\mathrm{CAD})(5,6)$. It is also clinically useful for characterization of high-risk plaque features, such as Napkin-ring sign (NRS), low attenuation plaque (LAP), spotty calcification and PR (7). Those highrisk features were found to be associated with unfavorable clinical outcomes or hemodynamic significance (8-14).

More recently, absolute plaque quantification analysis has been reported to be beneficial for improving risk stratification and dynamic follow-up of medical treatment $(15,16)$. With the technical development of CT hardware, dual-source CT (DSCT) equipped with fully integrated circuit detector system offers high spatial and temporal resolution (17). Given the fact that coronary plaques usually have very small volumes, this high-definition detector would theoretically benefit CT plaque evaluation by reducing partial volume effect especially for quantification of different intra-plaque components. We hypothesized that the plaque quantification by DSCT would be helpful for prediction of vulnerable plaques. Therefore, we aimed to study the diagnostic performance of quantified plaque analysis and high-risk plaque characterization by DSCT for identifying vulnerable plaques as determined by IVUS.

\section{Methods}

\section{Patient population}

Between January 2012 to December 2016, consecutive patients, who had stable angina (defined as Canadian Cardiovascular Society classification grade I to grade II) and referred for CCTA and IVUS, were retrospectively searched and reviewed. IVUS was clinically indicated by interventional cardiologists during the procedure of invasive coronary angiography (ICA) after CCTA to evaluate the morphological features of intermediate lesions in order to determine optimal treatment strategy. The inclusion criteria were: (I) CCTA and IVUS were performed to assess native coronary lesions within 4 weeks; (II) the image quality of CCTA was excellent or good (without artifact or with mild artifact). Exclusion criteria were as follows: (I) CCTA and IVUS were performed to evaluate in-stent restenosis; (II) the image quality of CCTA was sufficient or poor (with moderate or severe artifact); (III) the interval between CCTA and IVUS was more than 4 weeks (Figure 1). Hospital ethic committee approved this retrospective study and informed consent was waived from all patients.

\section{CCTA protocol}

A second-generation DSCT (Somatom Definition FLASH, Siemens Healthineers) equipped with fully integrated circuit detector was employed for scanning. Nitroglycerin $(0.5 \mathrm{mg})$ was given sublingually in all patients whereas betablocker was not administrated. Calcium score scan was first performed to calculate the Agatston score of coronary arteries. After then, a bolus of 60 to $70 \mathrm{~mL}$ contrast media (iopamidol $370 \mathrm{mg}$ iodine $/ \mathrm{mL}$, Bayer) was injected into the antecubital vein at the rate of $4.5-5 \mathrm{~mL} / \mathrm{s}$, followed by injection of a $20-40 \mathrm{~mL}$ saline flush. Bolus tracking was used with automated study triggering when the attenuation reached $100 \mathrm{HU}$ in a region of interest within the ascending aorta with a 6 seconds delay. Prospective ECG-triggered sequential acquisition was performed in all patients (with the coverage of $30 \%$ to $80 \%$ of R-R interval) and the detailed scanning parameters were listed as follow: collimation $=64 \times 0.6 \mathrm{~mm}$, reconstructed slice thickness $=$ $0.75 \mathrm{~mm}$, reconstructed slice interval $=0.5 \mathrm{~mm}$, rotation 
time $=280 \mathrm{~ms}$ and application of automated tube voltage and current modulation (CAREKv, CAREDose 4D, Siemens Healthineers).

\section{CT-derived plaque analysis}

All data were reconstructed with a medium soft kernel (I26f) and iterative reconstruction technique (SAFIRE, Siemens Healthineers). Datasets of all available cardiac phases were then transferred to an offline workstation (Syngo.Via, Siemens Healthineers) and the phases with best image quality were manually selected for further analysis. The image quality was assessed by 4-grade scale: excellent (absence of artifact); good (presence of mild artifact), sufficient (presence of moderate artifact, but still diagnostic), poor (presence of severe artifact, non-diagnostic). Only patients with image quality of excellent and good were included for further analysis. The image quality of CCTA was assessed by various objective image quality parameters (18). The details of image quality evaluation were given in supplementary.

All lesions with CCTA and IVUS validation were analyzed using a dedicated plaque analysis software (Coronary Plaque Analysis, version 2.0, Siemens Healthineers). This software enables quantification of different plaque components based on HU thresholds. The borders of target lesions were semi-automatically delineated once the proximal and distal shoulders of the plaques were determined. Manual adjustment of plaque borders was needed when necessary. The measured parameters were listed as follow: (I) the minimal lumen area (MLA) was measured manually with a digital caliper at the narrowest level of the lesion on the cross-sectional images; (II) the diameter stenosis (DS) was defined as (reference diameter - minimal lumen diameter)/reference diameter; (III) the plaque burden was defined as (vessel cross-sectional area MLA)/cross-sectional area; (IV) the remodeling index (RI) was defined as the ratio of cross-sectional vessel area of the lesion to the proximal reference area and PR was defined as a $\mathrm{RI} \geq 1.1(7)$; (V) LAP was defined as any voxel $<30 \mathrm{HU}$ within a coronary plaque (7) and total plaque volume (TPV) as well as LAP volume were quantified by the dedicated plaque analysis software; (VI) spotty calcification was defined by an intra-lesion calcific plaque $<3 \mathrm{~mm}$ in length that comprised $<90$ degrees of the lesion circumference (7); (VII) NRS was characterized by a plaque core with low computed tomography attenuation surrounded by a rimlike area of higher attenuation as previous reported (8).
Calcified lesions were not excluded from analysis regardless of calcium burden. In order to ensure that identical lesions were assessed by CCTA and IVUS, anatomical landmarks, such as coronary ostium and bifurcation were used for reference. In brief, the distance between target lesions and anatomical landmarks was measured by IVUS and the same distance distal to the landmarks was used to locate the plaque on CCTA.

The image quality of CCTA and all plaque characterization was retrospectively evaluated by two cardiovascular radiologists (with 10 and 9 years of experience on cardiac imaging), who were blinded to ICA and IVUS results. The mean values of quantitative parameters measured by two observers were used for further analysis.

\section{ICA procedure and IVUS analysis}

The ICA was performed with standard techniques, and at least 2 different views were obtained for each main vessel. Significant coronary stenosis was defined as luminal stenosis $\geq 50 \%$ by visual assessment. All segments were retrospectively evaluated by two interventional cardiologists (with 16 and 12 years of experience on coronary intervention), who were blinded to the results of CCTA.

The IVUS and virtual histology IVUS (VH-IVUS) were acquired with 3.2 F $20 \mathrm{MHz}$ Eagle-eye Gold catheters (Volcano) using a motorized pull-back at $1 \mathrm{~mm} / \mathrm{s}$. The IVUS images were digitally recorded and archived. IVUS and VH-IVUS images were retrospectively analyzed by same two interventional cardiologists with the INDEC Echo Plaque software (INDEC Medical System). TCFA lesions were defined as plaque burden $>40 \%$, confluent necrotic core $>10 \%$ plaque cross-sectional area, in contact with vessel lumen for 3 consecutive frames (19). Any disagreement was resolved by means of consensus.

\section{Statistical analysis}

Statistical analysis was performed using commercially available statistical software (MedCalc Statistical Software version 15.2.2, MedCalc Software bvba and SPSS Statistical Software version 17.0). One-sample KolmogorovSmirnov test was used to check the assumption of normal distribution. Quantitative variables with normal distribution were expressed as means \pm standard deviations while median and quartiles were used otherwise. Categorical variables were reported as count (\%), and compared by the Fisher's 
Table 1 Clinical characteristics

\begin{tabular}{|c|c|}
\hline Characteristic & Datum \\
\hline Number of patients & 65 \\
\hline Number of lesions & 89 \\
\hline Ages (years) ${ }^{a}$ & $69.8 \pm 9.2$ \\
\hline Male & $36(55.4 \%)$ \\
\hline \multicolumn{2}{|l|}{ Risk factors ${ }^{\mathrm{b}}$} \\
\hline Hypertension & $52(80.0 \%)$ \\
\hline Diabetes mellitus & $12(18.5 \%)$ \\
\hline Dyslipidemia & $11(16.9 \%)$ \\
\hline Current smoker & $17(26.2 \%)$ \\
\hline \multicolumn{2}{|l|}{ Distribution of lesion ${ }^{\mathrm{b}}$} \\
\hline Left main coronary artery & $2(2.2 \%)$ \\
\hline Left anterior descending & $50(56.2 \%)$ \\
\hline Right coronary artery & $25(28.1 \%)$ \\
\hline Left circumflex artery & $10(11.2 \%)$ \\
\hline Diagonal branch & $1(1.1 \%)$ \\
\hline Obtuse marginal & $1(1.1 \%)$ \\
\hline
\end{tabular}

Unless otherwise specified, data are numbers of patients with percentages in parentheses. ${ }^{\text {a }}$, data are mean \pm the standard deviation; ${ }^{b}$, data are numbers of lesions, with percentages in parentheses.

exact test or chi-square test, according to the data cell size. Student's $\mathrm{t}$ test was used for normally distributed data, and the Mann-Whitney $\mathrm{U}$ test was used for data that were not normally distributed. Variables that were significant at univariate analysis were included for further multivariate analysis. Multivariate regression analysis was used to determine the independent predictors of IVUS-determined TCFA lesions which were performed using the "forward" method. The model included variables with $\mathrm{P}$ value $<0.10$ in the univariate regression analysis. Inter-observer agreements of all parameters were examined for intra-class correlation coefficients (ICC). A two-tailed $\mathrm{P}$ value $<0.05$ was statistically considered significant.

\section{Results}

\section{Clinical characteristics}

A total of 84 patients undergoing both CCTA and IVUS were initially reviewed. Four patients were excluded because the interval between CCTA and ICA was longer than 4 weeks whereas another exclusion of 8 patients was due to significantly impaired CCTA image quality. Seven patients with previous revascularization of target lesions were further excluded (Figure 1). Finally, 65 patients (mean age: 69.8 \pm 9.2 (range, $51-86$ ) years; 36 males (mean age: 67.6 \pm 9.5 years; range, $51-84$ years, and 29 females (mean age: $72.5 \pm$ 8.2 years; range $51-86$ years) with 89 lesions were included in our study. The mean interval between CCTA and IVUS was $9.3 \pm 4.7$ days (range, 1 to 25 days). The mean effective dose was $4.2 \pm 1.5$ (range, $2.3-7.6$ ) $\mathrm{mSv}$. The percentages of patients scanned with 80,100 and $120 \mathrm{kV}$ were $36.9 \%$ (24/65), 50.8\% (33/65) and $12.3 \%(8 / 65)$ respectively. The mean time for plaque quantification was $12.8 \pm 4.7$ minutes. None of the target lesions had predilatation before IVUS imaging. Detailed demographic data was given in Table 1.

\section{Correlation of CT-derived quantitative plaque parameters with TCFAs determined by IVUS}

The inter-observer agreement for various CT-derived quantitative and qualitative parameters was excellent (Table S1).The image quality of CCTA was also listed in Table S2. LAP and NRS were more frequently presented in the group of TCFA lesions than in the group of nonTCFA lesions ( $\mathrm{P} \leq \mathrm{RS}$ w0 for both) (Table 2). The mean LAP volume of TCFA lesions was significantly larger than that of non-TCFA lesions [16.5 (11.0-23.0) vs. $0(0-1.5) \mathrm{mm}^{3}$, $\mathrm{P}<0.001$, Figure 2]. In contrast, there was no significant difference between group of TCFA lesions and nonTCFA lesions with respect to any other CT parameters, such as DS, MLA, plaque burden, spotty calcification, PR, remodeling index and TPV $(\mathrm{P}>0.05$ for all) (Table 2).

\section{Diagnostic performance of CT-derived quantitative plaque parameters for predicting TCFAs}

In univariate analysis, LAP, NRS, and LAP volume were all significant predictors for TCFA lesions (details in Table 3). According to multivariate logistic regression analysis, only LAP volume remained significant predictor for IVUSconfirmed vulnerable plaques (odds ratio $=3.294,95 \%$ confidence interval: 1.177-9.223, $\mathrm{P}=0.023)$.

According to ROC curve analysis, LAP volume showed largest AUC for diagnosing TCFA lesions (AUC $=0.901$, 95\% confidence interval: 0.819-0.954, $\mathrm{P}<0.0001$ ) (Figure 3). When using $>8 \mathrm{~mm}^{3}$ as the best cutoff value, the diagnostic accuracy, sensitivity and specificity of LAP volume for 
Table 2 Comparison of CT-derived plaque parameters between TCFA and non-TCFA lesions

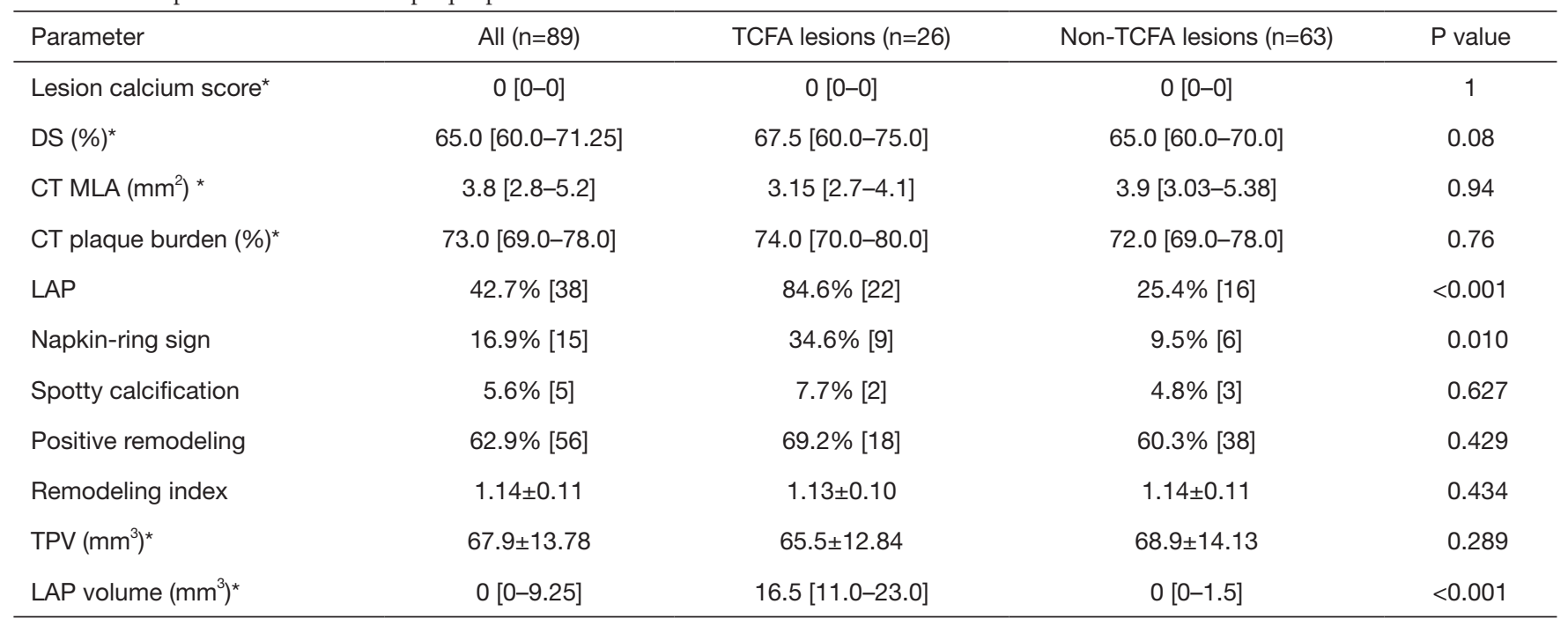

${ }^{*}$ Results are shown as median (1st quartile to 3rd quartile). CT, computed tomography; DS, diameter stenosis; LAP, low attenuation plaque; MLA, minimal lumen area; TCFA, thin-cap fibroatheroma; TPV, total plaque volume.

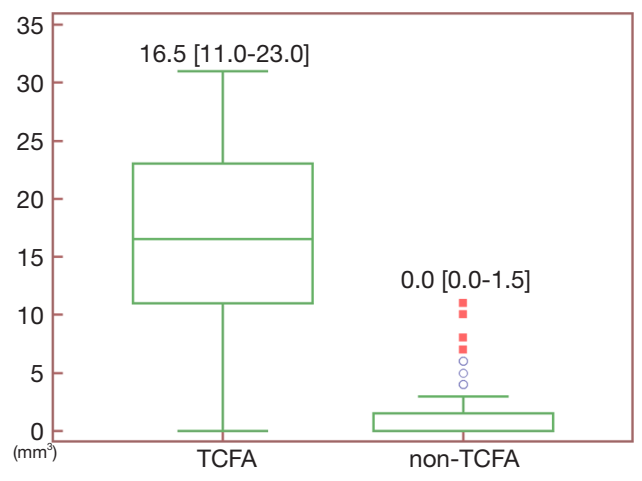

Figure 2 Box plot showing the difference of LAP volume between TCFA and non-TCFA lesions. The LAP volume of TCFAs is significantly larger than that of non-TCFAs [16.5 (11.0-23.0) vs. 0 $\left.(0-1.5) \mathrm{mm}^{3}, \mathrm{P}<0.001\right]$. LAP, low attenuation plaque; TCFA, thincap fibroatheroma.

predicting TCFA lesions were 91.0\% (81/89), 84.6\% $(22 / 26)$ and $96.8 \%(61 / 63)$ respectively. Representative cases were given in Figures 4 and 5.

\section{Discussion}

The major finding of the current study was that LAP volume was significantly larger in TCFA lesions and this parameter was the strongest predictors for vulnerable plaques.
Coronary vulnerable plaque, or TCFA, is pathologically characterized by large necrotic core covered by thin fibrous cap (2). TCFAs are prone to progress or even rupture under the effect of low endothelial shear stress $(20,21)$. CCTA is currently the best non-invasive imaging modality for morphological assessment of coronary plaques (22). According to previous ex vivo human hearts studies, CCTA plaque characterization showed promising results to predict advanced atheroma with reference to histologic validation (23-25). However, these studies did not take the quantitative data, such as TPV and LAP volume, into account. Due to the limited spatial resolution (in-plane resolution of 300 to $400 \mu \mathrm{m})$ of CT detectors, it is not technically feasible to directly visualize the thin fibrous cap $(<65 \mu \mathrm{m})$ of TCFAs. Thus, traditional high-risk plaque features are only indirect signs and may not be enough to predict TCFAs.

In line with our initial hypothesis, the absolute LAP volume outperformed other traditional high-risk plaque features for prediction of TCFA lesions. This could be ascribed to the following reasons. First, LAP is a qualitative description of the presence of any intra-plaque voxel with attenuation value $<30 \mathrm{HU}$. Nevertheless, according to the diagnostic criteria for TCFA lesions by IVUS, the confluent necrotic core should account for at least $10 \%$ of plaque cross-sectional area (19). In other words, the LAP volume characterized by CT has to be "big" enough to correlate to the TCFA lesions as determined by VH-IVUS. According to the current results, the best cut-off value of LAP volume 
Table 3 Univariate and multivariate analysis: CT-derived plaque analysis for detecting TCFA lesions

\begin{tabular}{|c|c|c|c|c|c|c|}
\hline Variable & \multicolumn{3}{|c|}{ Univariate analysis } & \multicolumn{3}{|c|}{ Multivariate analysis } \\
\hline DS (\%) & 0.764 & $0.562-1.038$ & 0.085 & 0.673 & $0.336-1.349$ & 0.264 \\
\hline CT MLA $\left(\mathrm{mm}^{2}\right)$ & 0.964 & $0.374-2.484$ & 0.939 & & & \\
\hline CT plaque burden (\%) & 0.994 & $0.955-1.034$ & 0.758 & & & \\
\hline Napkin-ring sign & 5.029 & $1.567-16.146$ & 0.007 & 4.801 & $0.586-39.352$ & 0.144 \\
\hline Spotty calcification & 1.667 & $0.262-10.608$ & 0.589 & & & \\
\hline Positive remodeling & 1.480 & $0.559-3.920$ & 0.430 & & & \\
\hline Remodeling index & 0.534 & $0.008-35.208$ & 0.769 & & & \\
\hline
\end{tabular}

CT, computed tomography; DS, diameter stenosis; LAP, low attenuation plaque; MLA, minimal lumen area; TCFA, thin-cap fibroatheroma; TPV, total plaque volume.

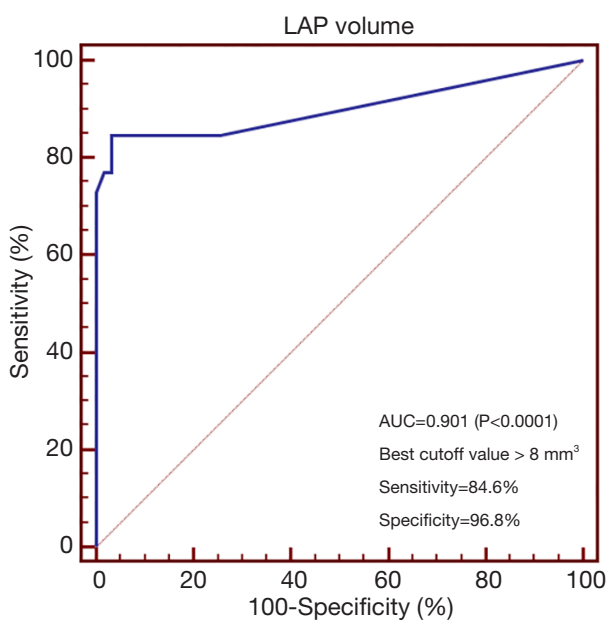

Figure 3 ROC curve analysis of LAP volume for identification of TCFA lesions. AUC, area under curve; LAP, low attenuation plaque; ROC, receiver operating characteristic.

to predict TCFA lesions was $8 \mathrm{~mm}^{3}$. Considering the fact that mean TPV of TCFA group was $65.5 \mathrm{~mm}^{3}, 8 \mathrm{~mm}^{3}$ accounted for approximately $12.2 \%$ of TPV. Interestingly, this number is similar to the diagnostic criteria for TCFAs by VH-IVUS. Therefore, it is conceivable that the absolute quantification of LAP volume was more accurate than LAP alone to predict TCFA lesions. Second, the identification of traditional high-risk plaque features, such as NRS, is relatively a subjective diagnosis and can sometimes be equivocal. In contrast, the quantification of LAP volume is an objective measurement and more reproducible than the recognition of those traditional high-risk plaque features. Third, NRS is considered to be related with neoangiogenesis of vasa vasorum, which is secondary to plaque inflammation in vulnerable plaques (26). However, NRS is originally discovered for culprit lesions in patients with ACS $(8,27,28)$. The incidence of NRS varied significantly between different cohorts, especially for patients with ACS or with stable angina. One study by Tomizawa showed that NRS was observed in $77 \%$ of TCFA lesions and the overall incidence of NRS in that cohort was $52 \%$ (29). Another study with larger sample size only revealed the presence of NRS in $1.4 \%$ of all population (30). The current study demonstrated that NRS was presented in $34.6 \%$ of TCFA lesions and $9.5 \%$ of non-TCFA lesions and overall incidence of NRS was $16.9 \%$. The heterogeneity of different cohorts, as noted in different studies, might be the main reason for such varied incidence of NRS. Due to the above factors, it is reasonable for absolute LAP volume to outperform other parameters to predict TCFAs.

The potential clinical implication of the present results lies in the use of quantified LAP volume to non-invasively screen for vulnerable coronary plaques. Previous studies with optical coherence tomography (OCT) as the reference standard have confirmed the value of CCTA-derived highrisk plaque features for detection of TCFA lesion (31-33). However, only qualitative parameters, such as PR, presence 


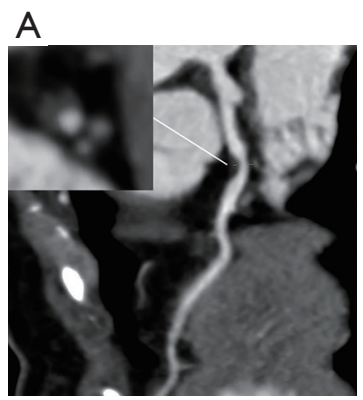

B

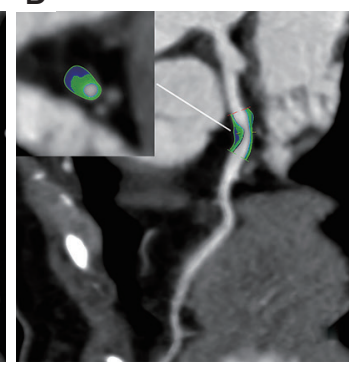

C

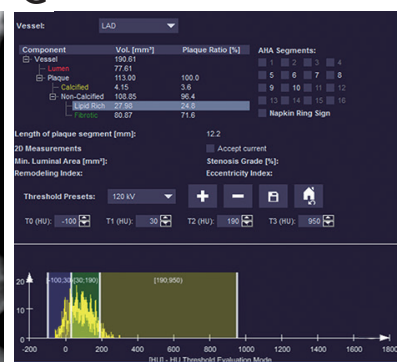

D

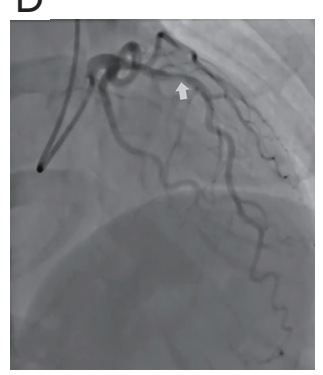

$\mathrm{E}$

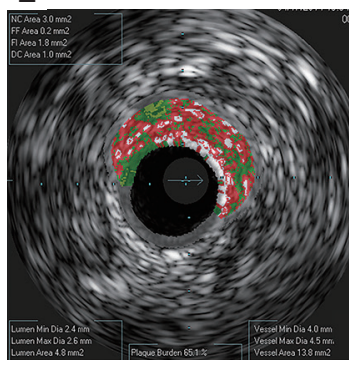

Figure 4 Representative case of CCTA plaque quantification in patient with TCFA lesion. (A) Curved planar reformation image showed a non-calcified plaque at proximal LAD with moderate stenosis. (B) The color-coded CPR image using a dedicated plaque analysis software identified the presence of low attenuation components (labeled as blue, -100 to $30 \mathrm{HU}$ ) within the plaque. (C) Histogram of different plaque components based on $\mathrm{HU}$ thresholds. The total plaque volume was $113 \mathrm{~mm}^{3}$ while the low attenuation component volume was $27.98 \mathrm{~mm}^{3}$. (D) ICA showed intermediate coronary stenosis (white arrow) at the proximal LAD. (E) VH-IVUS confirmed the diagnosis of TCFA lesion, showing large necrotic core (labelled as red) within the plaque. CCTA, coronary computed tomography angiography; ICA, invasive coronary angiography; LAD, left anterior descending; TCFA, thin-cap fibroatheroma; VH-IVUS, virtual histology intravascular ultrasound.

A

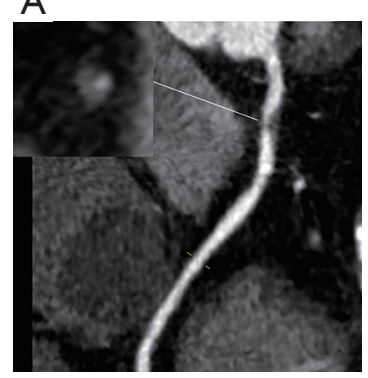

B

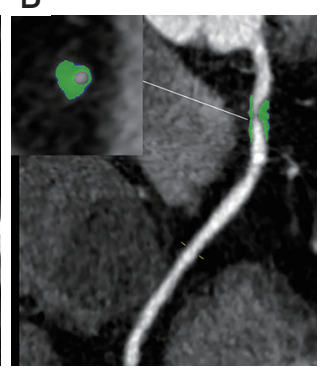

C

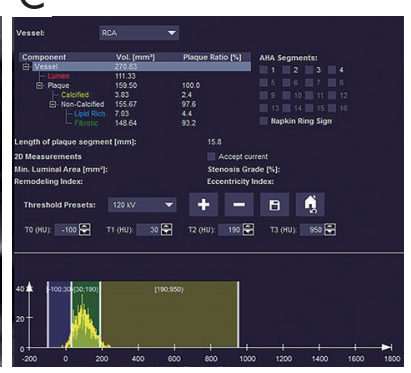

$\mathrm{D}$

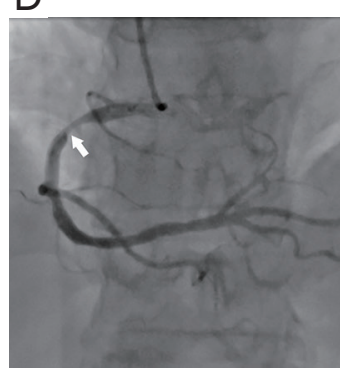

$\mathrm{E}$

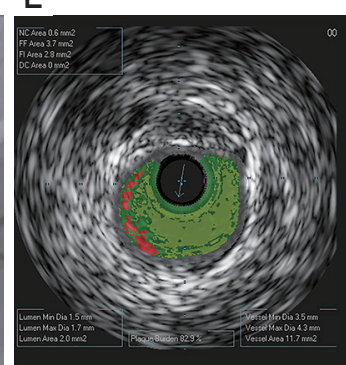

Figure 5 Representative case of CCTA plaque quantification in patient with non-TCFA lesion. (A) Curved planar reformation image showed a non-calcified plaque at proximal RCA with severe stenosis. (B) The color-coded CPR image using a dedicated plaque analysis software revealed minimal presence of LAP component within the plaque. (C) Histogram of different plaque components based on HU thresholds. The total plaque volume was $159.5 \mathrm{~mm}^{3}$ while the low attenuation component volume was $7.03 \mathrm{~mm}^{3}$. (D) ICA showed severe coronary stenosis (white arrow) at the proximal RCA. (E) VH-IVUS confirmed the diagnosis of non-TCFA lesion, showing minimal necrotic core (labelled as red) within the plaque. CCTA, coronary computed tomography angiography; ICA, invasive coronary angiography; LAP, low attenuation plaque; RCA, right coronary artery; TCFA, thin-cap fibroatheroma; VH-IVUS, virtual histology intravascular ultrasound.

of LAP and NRS, were used in those studies. In contrast, the current study validated the quantified LAP volume as the strongest predictor of TCFAs and using the cutoff value of $8 \mathrm{~mm}^{3}$ outperformed other qualitative parameters for identifying TCFAs. Therefore, CT-based plaque quantitative analysis might be more helpful to identify the subgroup of high-risk intermediate stenosis. In that case, intensified medical treatment may be warranted in order to stabilize the lesions and CCTA can also serve as the ideal follow-up imaging modality to monitor the change of plaque composition (16).
It is of note that the clinical outcome data of the current cohort was not available due to the retrospective design of the study. Other previous studies have revealed the prognostic implications of CT-derived plaque features, such as conventional high-risk plaque features and quantified lipid rich necrotic core volume (34-37). Although we validated LAP volume as a strong independent predictor for TCFA lesions, it still needs to be verified whether LAP volume also carries prognostic value like other conventional high-risk plaque features have.

Despite of the above promising results, the current study 
has several limitations. First, IVUS was clinically indicated in the present cohort to evaluate the intermediate lesions. This leaded to inclusion bias because very few target lesions in the current study had mild or severe stenosis. Therefore, the imaging findings of TCFAs in mild or severe stenosis still need to be explored. Second, OCT, which is considered the gold-standard for in vivo imaging of vulnerable plaques, was not available for identification of TCFAs in the current study (38). Since IVUS has lower spatial resolution as compared to OCT to detect TCFAs (38), the present findings still need to be confirmed by future studies with reference to OCT. Finally, CT plaque images were acquired by a second generation DSCT using varied $\mathrm{kV}$ settings in different patients. As shown by one previous study, different tube voltages might make minor differences in quantification of plaque attenuation (39). Therefore, future studies with more homogeneous acquisition parameters are warranted to confirm the present findings.

In conclusion, CT-derived LAP volume of TCFA lesions was significantly higher than those of non-TCFA lesions. LAP volume was the strongest predictor for TCFA lesions as validated by IVUS.

\section{Acknowledgments}

Funding: This study is supported by 2017 People's Livelihood Project of Pudong Committee on Science and Technology in Shanghai (No. PKJ2017-Y39), Key and Weak Specialty Construction Program of Pudong Health Bureau of Shanghai (No. PWZbr2017-11), Innovative And Collaborative Project Funding of Shanghai University of Medicine and Health Sciences (No. SPCI-18-17-001) and 2017 General Research Projects of Health Bureau of Shanghai (No. 201740199).

\section{Footnote}

Conflicts of Interest: The authors have no conflicts of interest to declare.

Ethical Statement: Hospital ethic committee approved this retrospective study and informed consent was waived from all patients.

Open Access Statement: This is an Open Access article distributed in accordance with the Creative Commons Attribution-NonCommercial-NoDerivs 4.0 International License (CC BY-NC-ND 4.0), which permits the non- commercial replication and distribution of the article with the strict proviso that no changes or edits are made and the original work is properly cited (including links to both the formal publication through the relevant DOI and the license). See: https://creativecommons.org/licenses/by-nc-nd/4.0/.

\section{References}

1. Arbab-Zadeh A, Fuster V. The myth of the "vulnerable plaque": transitioning from a focus on individual lesions to atherosclerotic disease burden for coronary artery disease risk assessment. J Am Coll Cardiol 2015;65:846-55.

2. Toutouzas K, Benetos G, Karanasos A, Chatzizisis YS, Giannopoulos AA, Tousoulis D. Vulnerable plaque imaging: updates on new pathobiological mechanisms. Eur Heart J 2015;36:3147-54.

3. Falk E, Nakano M, Bentzon JF, Finn AV, Virmani R. Update on acute coronary syndromes: the pathologists' view. Eur Heart J 2013;34:719-28.

4. Stone GW, Maehara A, Lansky AJ, de Bruyne B, Cristea E, Mintz GS, Mehran R, McPherson J, Farhat N, Marso SP, Parise H, Templin B, White R, Zhang Z, Serruys PW; PROSPECT Investigators. A prospective naturalhistory study of coronary atherosclerosis. N Engl J Med 2011;364:226-35.

5. Meijboom WB, Meijs MF, Schuijf JD, Cramer MJ, Mollet NR, van Mieghem CA, Nieman K, van Werkhoven JM, Pundziute G, Weustink AC, de Vos AM, Pugliese F, Rensing B, Jukema JW, Bax JJ, Prokop M, Doevendans PA, Hunink MG, Krestin GP, de Feyter PJ. Diagnostic accuracy of 64-slice computed tomography coronary angiography: a prospective, multicenter, multivendor study. J Am Coll Cardiol 2008;52:2135-44.

6. Miller JM, Rochitte CE, Dewey M, Arbab-Zadeh A, Niinuma H, Gottlieb I, Paul N, Clouse ME, Shapiro EP, Hoe J, Lardo AC, Bush DE, de Roos A, Cox C, Brinker J, Lima JA. Diagnostic performance of coronary angiography by 64-row CT. N Engl J Med 2008;359:2324-36.

7. Motoyama S, Sarai M, Harigaya H, Anno H, Inoue K, Hara T, Naruse H, Ishii J, Hishida H, Wong ND, Virmani R, Kondo T, Ozaki Y, Narula J. Computed tomographic angiography characteristics of atherosclerotic plaques subsequently resulting in acute coronary syndrome. J Am Coll Cardiol 2009;54:49-57.

8. Otsuka K, Fukuda S, Tanaka A, Nakanishi K, Taguchi H, Yoshikawa J, Shimada K, Yoshiyama M. Napkin-ring sign on coronary CT angiography for the prediction of acute coronary syndrome. JACC Cardiovasc Imaging 
2013;6:448-57.

9. Williams MC, Moss AJ, Dweck M, Adamson PD, Alam S, Hunter A, Shah ASV, Pawade T, Weir-McCall JR, Roditi G, van Beek EJR, Newby DE, Nicol ED. Coronary Artery Plaque Characteristics Associated With Adverse Outcomes in the SCOT-HEART Study. J Am Coll Cardiol 2019;73:291-301.

10. Yu M, Li W, Lu Z, Wei M, Yan J, Zhang J. Quantitative baseline CT plaque characterization of unrevascularized non-culprit intermediate coronary stenosis predicts lesion volume progression and long-term prognosis: A serial CT follow-up study. Int J Cardiol 2018;264:181-6.

11. Yu M, Lu Z, Li W, Wei M, Yan J, Zhang J. Coronary plaque characteristics on baseline CT predict the need for late revascularization in symptomatic patients after percutaneous intervention. Eur Radiol 2018;28:3441-53.

12. Yu M, Lu Z, Shen C, Yan J, Wang Y, Lu B, Zhang J. The best predictor of ischemic coronary stenosis: subtended myocardial volume, machine learning-based FFRCT, or high-risk plaque features? Eur Radiol 2019;29:3647-57.

13. Foldyna B, Eslami P, Scholtz JE, Baltrusaitis K, Lu MT, Massaro JM, D'Agostino RB Sr, Ferencik M, Aerts HJWL, O'Donnell CJ, Hoffmann U. Density and morphology of coronary artery calcium for the prediction of cardiovascular events: insights from the Framingham Heart Study. Eur Radiol 2019;29:6140-8.

14. Park J, Lee JM, Koo BK, Choi G, Hwang D, Rhee TM, Yang S, Park J, Zhang J, Kim KJ, Tong Y, Doh JH, Nam CW, Shin ES, Cho YS, Chun EJ, Choi JH, Norgaard BL, Christiansen EH, Niemen K, Otake H, Penicka M, de Bruyne B, Kubo T, Akasaka T, Narula J, Douglas PS, Taylor CA. Relevance of anatomical, plaque, and hemodynamic characteristics of non-obstructive coronary lesions in the prediction of risk for acute coronary syndrome. Eur Radiol 2019;29:6119-28.

15. Lee SE, Sung JM, Rizvi A, Lin FY3, Kumar A, Hadamitzky M, Kim YJ, Conte E, Andreini D, Pontone G, Budoff MJ, Gottlieb I, Lee BK, Chun EJ, Cademartiri F, Maffei E, Marques H, Leipsic JA, Shin S, Hyun Choi J, Chinnaiyan K, Raff G, Virmani R, Samady H, Stone PH, Berman DS, Narula J, Shaw LJ, Bax JJ, Min JK, Chang HJ. Quantification of Coronary Atherosclerosis in the Assessment of Coronary Artery Disease. Circ Cardiovasc Imaging 2018;11:e007562.

16. Lee SE, Chang HJ, Sung JM, Park HB, Heo R, Rizvi A, Lin FY, Kumar A, Hadamitzky M, Kim YJ, Conte E, Andreini D, Pontone G, Budoff MJ, Gottlieb I, Lee BK, Chun EJ, Cademartiri F, Maffei E, Marques H, Leipsic
JA, Shin S, Choi JH, Chinnaiyan K, Raff G, Virmani R, Samady H, Stone PH, Berman DS, Narula J, Shaw LJ, Bax JJ, Min JK. Effects of Statins on Coronary Atherosclerotic Plaques: The PARADIGM Study. JACC Cardiovasc Imaging 2018;11:1475-84.

17. Morsbach F, Desbiolles L, Plass A, Leschka S, Schmidt B, Falk V, Alkadhi H, Stolzmann P. Stenosis quantification in coronary CT angiography: impact of an integrated circuit detector with iterative reconstruction. Invest Radiol 2013;48:32-40.

18. Li Y, Yu M, Li W, Lu Z, Wei M, Zhang J. Third generation dual-source CT enables accurate diagnosis of coronary restenosis in all size stents with low radiation dose and preserved image quality. Eur Radiol 2018;28:2647-54.

19. Calvert PA, Obaid DR, O'Sullivan M, Shapiro LM, McNab D, Densem CG, Schofield PM, Braganza D, Clarke SC, Ray KK, West NE, Bennett MR. Association between IVUS findingsand adverse outcomes in patients with coronary artery disease the VIVA (VH IVUS in vulnerable atherosclerosis) study. JACC Cardiovasc Imaging 2011;4:894-901.

20. Stone PH, Saito S, Takahashi S, Makita Y, Nakamura S, Kawasaki T, Takahashi A, Katsuki T, Nakamura S, Namiki A, Hirohata A, Matsumura T, Yamazaki S, Yokoi H, Tanaka S, Otsuji S, Yoshimachi F, Honye J, Harwood D, Reitman M, Coskun AU, Papafaklis MI, Feldman CL; PREDICTION Investigators. Prediction of progression of coronary artery disease and clinical outcomes using vascular profiling of endothelial shear stress and arterial plaque characteristics: the prediction study. Circulation 2012;126:172-81.

21. Wentzel JJ, Chatzizisis YS, Gijsen FJ, Giannoglou GD, Feldman CL, Stone PH. Endothelial shear stress in the evolution of coronary atherosclerotic plaque and vascular remodeling: current understanding and remaining questions. Cardiovasc Res 2012;96:234-43.

22. Yoo SM, Lee HY, Jin KN, Chun EJ, Ann AF, White CS. Current Concepts of Vulnerable Plaque on Coronary CT Angiography. Cardiovasc Imaging Asia 2017;1:4-12.

23. Maurovich-Horvat P, Schlett CL, Alkadhi H, Nakano M, Stolzmann P, Vorpahl M, Scheffel H, Tanaka A, Warger WC 2nd, Maehara A, Ma S, Kriegel MF, Kaple RK, Seifarth H, Bamberg F, Mintz GS, Tearney GJ, Virmani R, Hoffmann U. Differentiation of early from advanced coronary atherosclerotic lesions: systematic comparison of CT, intravascular US, and optical frequency domain imaging with histopathologic examination in ex vivo human hearts. Radiology 2012;265:393-401.

24. Maurovich-Horvat P, Schlett CL, Alkadhi H, Nakano M, Otsuka F, Stolzmann P, Scheffel H, Ferencik M, Kriegel MF, 
Seifarth H, Virmani R, Hoffmann U. The napkin-ring sign indicates advanced atherosclerotic lesions in coronary CT angiography. JACC Cardiovasc Imaging 2012;5:1243-52.

25. Seifarth H, Schlett CL, Nakano M, Otsuka F, Károlyi M, Liew G, Maurovich-Horvat P, Alkadhi H, Virmani R, Hoffmann U. Histopathological correlates of the napkin-ring sign plaque in coronary CT angiography. Atherosclerosis 2012;224:90-6.

26. Fleiner M, Kummer M, Mirlacher M, Sauter G, Cathomas G, Krapf R, Biedermann BC. Arterial neovascularization and inflammation in vulnerable patients: early and late signs of symptomatic atherosclerosis. Circulation 2004;110:2843-50.

27. Pflederer T, Marwan M, Schepis T, Ropers D, Seltmann M, Muschiol G, Daniel WG, Achenbach S. Characterization of culprit lesions in acute coronary syndromes using coronary dual-source CT angiography. Atherosclerosis 2010;211:437-44.

28. Nakazawa G, Tanabe K, Onuma Y, Yachi S, Aoki J, Yamamoto H, Higashikuni Y, Yagishita A, Nakajima H, Hara K. Efficacy of culprit plaque assessment by 64-slice multidetector computed tomography to predict transient no-reflow phenomenon during percutaneous coronary intervention. Am Heart J 2008;155:1150-7.

29. Tomizawa N, Yamamoto K, Inoh S, Nojo T, Nakamura S. Accuracy of computed tomography angiography to identify thin-cap fibroatheroma detected by optical coherence tomography. J Cardiovasc Comput Tomogr 2017;11:129-34.

30. Lee JM, Choi KH, Koo BK, Park J, Kim J, Hwang D, Rhee TM, Kim HY, Jung HW, Kim KJ, Yoshiaki K, Shin ES, Doh JH, Chang HJ, Cho YK, Yoon HJ, Nam CW, Hur SH, Wang J, Chen S, Kuramitsu S, Tanaka N, Matsuo H, Akasaka T. Prognostic Implications of Plaque Characteristics and Stenosis Severity in Patients With Coronary Artery Disease. J Am Coll Cardiol 2019;73:2413-24.

31. Kashiwagi M, Tanaka A, Kitabata H, Tsujioka H, Kataiwa H, Komukai K, Tanimoto T, Takemoto K, Takarada S, Kubo T, Hirata K, Nakamura N, Mizukoshi M, Imanishi T, Akasaka T. Feasibility of noninvasive assessment of thin-cap fibroatheroma by multidetector computed tomography. JACC Cardiovasc Imaging 2009;2:1412-9.

32. Soeda T, Uemura S, Morikawa Y, Ishigami K, Okayama S, Hee SJ, Nishida T, Onoue K, Somekawa S, Takeda Y, Kawata H, Horii M, Saito Y. Diagnostic accuracy of dualsource computed tomography in the characterization of coronary atherosclerotic plaques: comparison with intravascular optical coherence tomography. Int J Cardiol 2011;148:313-8.
33. Sato A, Hoshi T, Kakefuda Y, Hiraya D, Watabe H, Kawabe M, Akiyama D, Koike A, Aonuma K. In vivo evaluation of fibrous cap thickness by optical coherence tomography for positive remodeling and low-attenuation plaques assessed by computed tomography angiography. Int J Cardiol 2015;182:419-25.

34. van Assen M, Varga-Szemes A, Schoepf UJ, Duguay TM, Hudson HT, Egorova S, Johnson K, St Pierre S, Zaki B, Oudkerk M, Vliegenthart R, Buckler AJ. Automated plaque analysis for the prognostication of major adverse cardiac events. Eur J Radiol 2019;116:76-83.

35. Nance JW Jr, Schlett CL, Schoepf UJ, Oberoi S, Leisy HB, Barraza JM Jr, Headden GF, Nikolaou K, Bamberg F. Incremental prognostic value of different components of coronary atherosclerotic plaque at cardiac CT angiography beyond coronary calcification in patients with acute chest pain. Radiology 2012;264:679-90.

36. Tesche C, Plank F, De Cecco CN, Duguay TM, Albrecht MH, Varga-Szemes A, Bayer RR Nd, Yang J, Jacks IL, Gramer BM, Ebersberger U, Hoffmann E, Chiaramida SA, Feuchtner G, Schoepf UJ. Prognostic implications of coronary CT angiography-derived quantitative markers for the prediction of major adverse cardiac events. J Cardiovasc Comput Tomogr 2016;10:458-65.

37. Bauer RW, Thilo C, Chiaramida SA, Vogl TJ, Costello P, Schoepf UJ. Noncalcified atherosclerotic plaque burden at coronary CT angiography: a better predictor of ischemia at stress myocardial perfusion imaging than calcium score and stenosis severity. AJR Am J Roentgenol 2009;193:410-8.

38. Kubo T, Imanishi T, Takarada S, Kuroi A, Ueno S, Yamano T, Tanimoto T, Matsuo Y, Masho T, Kitabata H, Tsuda K, Tomobuchi Y, Akasaka T. Assessment of culprit lesion morphology in acute myocardial infarction: ability of optical coherence tomography compared with intravascular ultrasound and coronary angioscopy. J Am Coll Cardiol 2007;50:933-9.

39. Tanami Y, Ikeda E, Jinzaki M, Satoh K, Nishiwaki Y, Yamada M, Okada Y, Kuribayashi S. Computed tomographic attenuation value of coronary atherosclerotic plaques with different tube voltage: an ex vivo study. J Comput Assist Tomogr 2010;34:58-63.

Cite this article as: Yuan $\mathrm{M}, \mathrm{Wu} \mathrm{H}, \mathrm{Li} \mathrm{R}, \mathrm{Yu} M, \mathrm{Dai} X$ Zhang J. The value of quantified plaque analysis by dual-source coronary CT angiography to detect vulnerable plaques: a comparison study with intravascular ultrasound. Quant Imaging Med Surg 2020;10(3):668-677. doi: 10.21037/qims.2020.01.13 


\section{Supplementary}

\section{Coronary CT angiography image quality assessment}

Various objective image quality parameters, including image noise of the aortic root and proximal coronary arteries, signal-to-noise ratio (SNR), and contrast-to-noise ratio (CNR), were also evaluated. A circular region of interest (ROI) was manually placed in the aortic root at the level of the left main coronary artery ostium $(2.5 \mathrm{~cm}$ in diameter) to measure lumen attenuation and image noise. The same measurement was also performed in the proximal segments

Table S1 Interobserver reproducibility

\begin{tabular}{|c|c|c|c|}
\hline Variable & ICC & $95 \% \mathrm{Cl}$ & $P$ value \\
\hline DS & 0.86 & $0.812-0.906$ & $<0.001$ \\
\hline CT MLA & 0.82 & $0.775-0.894$ & $<0.001$ \\
\hline LAP & 0.91 & $0.887-0.934$ & $<0.001$ \\
\hline Napkin-ring sign & 0.93 & $0.891-0.972$ & $<0.001$ \\
\hline Spotty calcification & 0.95 & $0.903-0.974$ & $<0.001$ \\
\hline Positive remodeling & 0.89 & $0.856-0.962$ & $<0.001$ \\
\hline Remodeling index & 0.84 & $0.813-0.882$ & $<0.001$ \\
\hline TPV & 0.92 & $0.867-0.963$ & $<0.001$ \\
\hline LAP volume & 0.93 & $0.893-0.976$ & $<0.001$ \\
\hline
\end{tabular}

of the left and right coronary arteries (ROI was drowned as large as possible with exclusion of vessel wall). All ROIs were measured three times, and mean measurements were used for further analysis. The SNR of each measured vessel was calculated by dividing the mean $\mathrm{HU}$ by the mean image noise. The proximal vessel contrast was defined as the $\mathrm{HU}$ difference between the vessel lumen measurement and the measurement of surrounding tissue immediately next to the vessel contour. The CNR was calculated as the vessel contrast value divided by image noise of the corresponding vessel.

Table S2 Image quality of coronary CT angiography

\begin{tabular}{lccc}
\hline Vessel & Noise & CNR & SNR \\
\hline Aorta & $21.2 \pm 6.8$ & $27.9 \pm 5.6$ & $26.7 \pm 5.2$ \\
LM & $22.5 \pm 6.4$ & $27.3 \pm 6.7$ & $24.5 \pm 5.9$ \\
RCA & $24.3 \pm 5.8$ & $26.9 \pm 7.5$ & $24.2 \pm 6.3$ \\
LAD & $25.1 \pm 6.1$ & $27.7 \pm 6.5$ & $26.2 \pm 7.1$ \\
LCx & $27.9 \pm 7.8$ & $24.5 \pm 7.2$ & $22.3 \pm 6.9$ \\
\hline
\end{tabular}

CNR, contrast noise ratio, LAD, left anterior descending, LCx, left circumflex, LM, left main, RCA, right coronary artery, SNR, signal noise ratio. 